\title{
Neuromuscular blocking agents - when is the block deep enough? And how to safely reverse the neuromuscular blocker
}

\author{
Lines D \\ Department of Anaesthesia, Chris Hani Baragwanath Academic Hospital, University of the Witwatersrand \\ *Corresponding author, email: lines@pixie.co.za
}

Neuromuscular blocking agents have revolutionised the practice of anaesthesia and surgery. Before the introduction of these neuromuscular blockers into clinical practice, muscle paralysis was induced with high dose volatile anaesthesia with its attendant risks of respiratory and cardiac depression. Suxamethonium, d-tubocurarine and gallamine were generally available for clinical use by the late 1950s. It was after this that the modern concept of balanced anaesthesia was introduced into routine clinical practice.

Subsequent to this, many new neuromuscular blocking agents (NMBA) were synthesised with the ultimate quest of producing the ideal neuromuscular blockers (NMB).

The most recent addition in 2000 was rapacuronium which had a short onset of action similar to suxamethonium and a termination of action in 15 minutes. This drug came closer to the ideal NMB than any other drug before. Unfortunately, rapacuronium was withdrawn in 2001 because of reports of fatal bronchospasm. ${ }^{1}$ Sugammadex, a novel NMB reversal agent introduced into clinical practice in 2008 , is able to reverse rocuronium rapidly and from any depth of block. This combination provides an alternative to suxamethonium and satisfies many of the properties of an ideal NMBA.

\section{Trend in use of muscle relaxants pre- and post-sugammadex}

In 2010, Naguib et al. ${ }^{2}$ did an internet-based survey in the United States (USA) and Europe. The Anesthesia Patient Safety Foundation and the European Society of Anaesthesiology sent e-mails to their respective members with an attached questionnaire. The survey was performed to determine the current management of neuromuscular blockers in the USA and Europe. Even though the primary aim of the survey was to describe practice as pertains to reversal of neuromuscular blockers, interesting data was also collected as far as the use of modern day neuromuscular blockers was concerned. Suxamethonium was still being used in both Europe (85.8\%) and the USA (92.8\%) to facilitate tracheal intubation and rocuronium was the most common NMB used for surgical relaxation ( $72.9 \%$ in Europe and $89.6 \%$ in the USA). ${ }^{2}$ This survey was conducted at a time when sugammadex was not yet freely available in clinical practice. A total of 2636 questionnaires were returned for analysis. The response rate from the USA was $40.1 \%$ and Europe $15.06 \%$.
Despite the limitations that this survey had as result of a poor response rate, it does suggest that suxamethonium and rocuronium are the most common muscle relaxants used for tracheal intubation in environments where sugammadex is not freely available and suggests that rocuronium is probably the most widely used relaxant for surgical relaxation.

Since 2010, the landscape has changed considerably with the increasing availability of sugammadex. In environments where the use of sugammadex is not restricted, the use of rocuronium for intubation and surgical relaxation has most certainly increased and the use of suxamethonium, particularly in the emergency setting has decreased. In 2015 Ledowski et al. ${ }^{3}$ conducted a survey at the Royal Perth Hospital in Australia where the use of sugammadex was not restricted. This survey was done two and a half years after the introduction of sugammadex. The survey revealed that the majority of anaesthetists were using an amino-steroid (94.6\%) sugammadex (100\%) combination when paralysing patients. This showed a large increase in the use of an amino-steroid NMBA from 61\% in 2010 and also a large increase in sugammadex use from $10 \%$ in 2010 . Rocuronium was used in $88.7 \%$ of intubations, vecuronium in $5.6 \%$ of cases and suxamethonium in $2.8 \%$ of cases. Cis-atracurium and mivacurium were only used in $1.4 \%$ of cases. ${ }^{3}$ This survey was contextual as it was only done in one department in Australia, but it highlights the possible trend in use of muscle relaxants in environments where the use of sugammadex is not limited.

\section{How deep is deep enough?}

There can be no doubt that the use of muscle relaxants has allowed the anaesthetist to provide optimum surgical conditions in a variety of surgical procedures. Muscle relaxants allow the anaesthetist to employ a more balanced approach to anaesthesia thereby reducing the amount of volatile agents used which would otherwise be required to provide surgical relaxation. The popularity of laparoscopic abdominal procedures as well as the general availability of sugammadex has renewed the interest in the question as to whether deep neuromuscular blockade is required to improve not only surgical access but also to reduce surgical complications and improve patient outcomes in laparoscopic surgery. With the advent of sugammadex allowing rapid reversal of deep levels of muscle relaxation the question that is now been asked is "how much 
relaxant is enough?" Recently it has become common practice to employ deep neuromuscular blockade for the duration of the laparoscopic procedure until the fascia is closed before reversing with high dose sugammadex. Many laparoscopic surgeons are now also demanding a deep NMB (post-tetanic count 1-2) in all laparoscopic procedures necessitating the anaesthetist to use sugammadex for reversal of the block.

The benefits of deep neuromuscular block for the duration of laparoscopic procedures have centred on patient benefits and perceived improved surgical access. This has the potential to decrease surgical risk, decrease surgical time, decrease the conversion rate to an open procedure and have a patient benefit of decreasing postoperative pain and in particular shoulder pain and length of hospital stay. ${ }^{4}$ These finding are however not consistent with all studies. It is routine to create a pneumo-peritoneum with inflation pressure of $12-15 \mathrm{mmHg}$ to facilitate surgical access. The higher the inflation pressure the more physiologic disturbance (haemodynamic and respiratory) is caused and it is reported in some studies that decreasing inflation pressures to as low as $8 \mathrm{mmHg}$ may translate into less postoperative pain and good surgical access at a lower inflation pressure.

Laparoscopic surgery performed without muscle relaxation does require higher inflation pressures and results in unacceptable surgical conditions. In laparoscopic surgery done without neuromuscular block, sudden movements may occur in up to $30 \%$ of patients theoretically increasing the likelihood of complications. ${ }^{9}$ Evidence in support of deep NMB during laparoscopic surgery should focus on two aspects, the first being a patient outcome benefit and the second an improvement in surgical conditions.

Reversing NMB with neostigmine has limitations. A deep level of neuromuscular block cannot be reversed with neostigmine making the practice of deep NMB till the end of the procedure impractical. A train of four count (TOFC) of at least two twitches on the neuromuscular monitor is necessary before reversing with neostigmine. Adequate reversal may still take another 10-15 minutes to achieve a TOF ratio (TOFR) of 0.9 .

It has been estimated that it costs around 2.6 billion dollars to launch a new drug into clinical practice. ${ }^{5}$ Sugammadex comes at a significant financial cost and, considering that a dose of at least $4 \mathrm{mg} / \mathrm{Kg}$ is required to fully reverse a deep NMB from rocuronium, there needs to be undisputed evidence that it results in better patient outcomes and surgical conditions when compared to a well-managed moderate block which can be reversed with the less costly neostigmine, to justify its routine use. The acquisition cost of sugammadex is roughly 100 times that of neostigmine and this does become an important factor in cost constrained environments.

To attempt to answer this question The Acta Anaesthesiologica Scandinavica, in a publication in 2016, asked two sets of authors to debate the pros and cons of whether deep neuromuscular blockade is beneficial in laparoscopic surgery. The two authors agreed on a basic database of references to review evidence.
Each author's analysis and text was shared with the other authors during their preparation of manuscripts. Madsen et al. ${ }^{6}$ put forward a suggestion that there is data to support routine use of deep neuromuscular block for laparoscopic surgery whilst Kopman et al. ${ }^{7}$ argued that insufficient evidence exists.

Madsen et al. ${ }^{6}$ concede that the evidence for improved patient outcome is not clear, however one study by Martini et al. ${ }^{8}$ using secondary endpoints did show improved surgical conditions and thus a potential benefit.

Kopman et al. ${ }^{7}$ concluded that the evidence did not show sufficient benefit to the patient to justify the cost of using sugammadex to reverse deep neuromuscular blocks. They did, however, agree that the study by Martini et al. ${ }^{8}$ pointed to a benefit in terms of improving surgical conditions.

More recently Rosenberg et al. ${ }^{4}$ in 2017 looked specifically at whether deep neuromuscular block improved laparoscopic surgical conditions. They concluded that there was an improvement in laparoscopic surgical conditions when using sustained deep NMB and low inflation pressures. This difference was evident when they compared it to moderate NMB with low inflation pressures. However, when the comparison was made using moderate NMB with standard pressures, there was no improvement in surgical conditions. The conclusion in their study found greater benefit with the low inflation pressures compared with the depth of NMB was at least in part due to the insufflation pressure chosen for the investigation. The authors acknowledge that the low pressure group $(8 \mathrm{mmHg})$ may just be too low, and that comparisons need to be done with a greater range of insufflation pressures to really confirm the benefit of deep NMB for surgical conditions.

No studies are available that show a decrease in surgical complications as a result of maintaining deep NMB throughout the procedure. Surgical complications such as bowel perforation and gas emboli are rare and generally would occur at the time of trocar insertion. At this early stage in the procedure the initial bolus of relaxant used for intubation would still provide a moderate to deep level of relaxation.

Madsen et al. ${ }^{6}$ and Kopman et al. ${ }^{7}$ found only three papers that looked at patient outcomes. Two studies describing patients undergoing laparoscopic cholecystectomy found no decrease in postoperative pain ${ }^{9,10}$ while the third study showed a decrease in postoperative shoulder pain with deep levels of NMB and lower inflation pressures. ${ }^{11}$

In analysing both the pros and cons debates a number of confounding issues emerge which make it difficult to come to a conclusion as to whether deep neuromuscular block throughout the laparoscopic procedure is needed. This debate has common ground in that there is no argument that neuromuscular blockers are needed during laparoscopic surgery and that sugammadex is a better agent for NMB reversal than neostigmine. The debate centres on the issue of how much relaxation is necessary and whether having a deep level of NMB throughout the procedure, necessitating the routine use of sugammadex for reversal is justified in terms of overall patient and surgical benefit. 
There are no studies available at present clearly showing that maintaining deep NMB throughout laparoscopic surgery reduces surgical complications and only a limited number of articles suggest that there is a reduction in postoperative shoulder pain. ${ }^{9,10}$ Numerous other studies have shown an improvement in haemodynamic and respiratory impairment. ${ }^{12}$ A Cochrane review done by Gurusamy et al. in $2014^{13}$ suggested that low inflation pressures resulted in a reduction in shoulder pain postoperatively, however Rosenberg et al. ${ }^{4}$ in their randomised controlled study in 2017 found no difference in the incidence of shoulder pain postoperatively between patients where low inflation pressures $(8 \mathrm{mmHg})$ were employed compared to the patients who had standard inflation pressures $(12 \mathrm{mmHg})$. The Cochrane review done by Gurusamy ${ }^{13}$ also could not show a difference in the conversion rate to open cholecystectomy between the low pressure group and the standard pressure group. Length of hospital stay was statistically shorter in the low pressure group but was clinically not significant.

Surgeons may have an impression that deep NMB improves operating conditions. Studies done by Martini, ${ }^{6}$ Dubois $^{14}$ and Staehr-Rye ${ }^{10}$ looking at subjective ratings by the surgeon all showed a marginal to improved surgical conditions during deep NMB and low inflation pressures during laparoscopic procedures. In the Dubois study, ${ }^{14}$ it was found that in patients who were assigned to the shallow or moderate block group, at least half of the occurrences of unacceptable operating conditions occurred in patients with a TOFC of 4 . This represents at best a mild depth of block and it is not surprising that surgical conditions were unacceptable. Staehr-rye ${ }^{10}$ in their study reported that $40 \%$ of patients assigned to the deep NMB group had incidents of unacceptable surgical conditions. Rosenberg ${ }^{4}$ in a more recent study in 2017 also had patients in the deep NMB and low inflation pressure group who had unacceptable surgical conditions requiring intervention. Of importance is the fact that the depth of NMB is not the only factor determining adequacy of the surgical field. Individual variation, comorbidity, age, use of volatile agents, abdominal compartments previously stretched by a pregnancy, abdominal fat distribution, surgical skill and adequate inflation pressure may all play a part in overall surgical satisfaction at a particular depth of NMB.

Much of the disagreement in the pros and cons debate centres around the definitions of depth of NMBs, as well as the paucity of data of actual PTC and TOF measurements recorded at the time that the surgeon is dissatisfied with the operating conditions.

Had sugammadex not come with a large price tag, there would be no debate. Therefore, the question that needs to be answered is whether the cost of sugammadex is justified in the light of rather poor evidence suggesting a patient outcome benefit with deep NMB throughout laparoscopic surgery. While the evidence of deep NMB improving surgical conditions is more convincing, is it enough to justify the routine use of deep NMB throughout laparoscopic surgery necessitating the use of large doses of sugammadex?

The elephant in the room which has not been discussed in this debate is the incidence of postoperative residual curarisation after laparoscopic surgery irrespective of whether a moderate or deep NMB is used and what if any negative consequences can be shown. The average time for reversal of a moderate neuromuscular block is 2.7 minutes after administration of 2 mg.kg-1 sugammadex compared to 17.9 minutes after administration of $50 \mu \mathrm{g} . \mathrm{kg}-1$ neostigmine. ${ }^{15}$ Tiberiu et al. in 2015 did a retrospective study on 119 charts looking at residual curarisation and postoperative respiratory complications following laparoscopic sleeve gastrectomy. There was no significant difference between the use of sugammadex or neostigmine for reversal. ${ }^{16}$

Boon et al. $^{15}$ in a recent review from 2018 presented evidence showing that neuromuscular block reversal with sugammadex reduced the rate of postoperative residual curarisation (PORC) and pulmonary outcome compared to reversal with neostigmine. The studies show evidence that neuromuscular block reversal with sugammadex reduced the rate of PORC compared to reversal with neostigmine. In the study by Della Rocca et al. ${ }^{17}$ in 2014 a TOF ratio $<0.9$ at 5, 10, and 20 minutes after reversal agent was more frequent in patients receiving neostigmine than sugammadex. In the sugammadex group, no patients had a TOF ratio $<0.9$ after 20 minutes from sugammadex reversal irrespective of the depth of the block. In the neostigmine group, $18 \%$ and $66 \%$ of patients in the shallow and deep block groups, respectively, exhibited TOF ratio $<0.9$ at 20 minutes after administration of neostigmine. A $4 \%$ incidence of residual curarisation after reversal with sugammadex in unmonitored environments has been shown. ${ }^{17}$ This reinforces the need for neuromuscular monitoring even with the use of sugammadex.

Studies comparing sugammadex and neostigmine on incidence of postoperative residual curarisation and pulmonary outcome

\begin{tabular}{|c|c|c|c|c|c|c|}
\hline Author & Year & Design & Comparison & Monitoring & PORC & Pulmonary outcome \\
\hline Kotake & 2013 & $\begin{array}{l}\text { Prospective } \\
\text { observational }\end{array}$ & Sugammadex versus neostigmine & No & $4.3 \%$ versus $23.9 \%$ ** & UA \\
\hline Ledowski & 2014 & $\begin{array}{l}\text { Retrospective } \\
\text { cohort }\end{array}$ & Sugammadex versus neostigmine & Available & UA & $\begin{array}{l}\text { Reduced pulmonary } \\
\text { outcome score in ASA } \\
3-4 \text { patients ** }\end{array}$ \\
\hline \multirow[t]{2}{*}{ Brueckmann } & 2015 & RCT & Sugammadex versus neostigmine & Available & $0 \%$ versus $43.3 \% * *$ & $\begin{array}{c}\text { Respiratory disorders: } \\
1.4 \% \text { versus } 6.5 \% \#\end{array}$ \\
\hline & & & & & & $\begin{array}{c}\text { Hypoxemia: } 1.4 \% \text { versus } \\
2.6 \% \#\end{array}$ \\
\hline Boon & 2016 & RCT & Sugammadex versus neostigmine & No & $4 \%$ versus $70 \% * *$ & $\begin{array}{l}\text { Lowest } \mathrm{O}_{2} \text { saturation: } \\
93.3 \text { versus } 96.8 \%^{* *}\end{array}$ \\
\hline Nemes & 2017 & RCT & Sugammadex versus neostigmine & No & $3.7 \%$ versus $15.4 \%$ \# & UA \\
\hline
\end{tabular}

ASA, American Society of Anesthesiologists; PORC, postoperative residual curarization (train of four [TOF] ratio $<0.9$ after extubation); RCT, randomised controlled trial; UA, unavailable.* $p<0.05$. ${ }^{*} p<0.05 ;{ }^{* *} p<0.001: \# p>0.05^{15}$ 


\section{Reversal of NMBA}

Recovery from a non-depolarising muscle blocking agent (NDMBA) is initially due to redistribution from the acetylcholine (Ach) receptor into the plasma and then eliminated either by the liver or kidney or by other mechanisms such as ester-hydrolysis or Hoffman elimination. When repeated or high doses are given resulting in high receptor occupancy, the reversal becomes more dependent on elimination. Recovery from NDMBA is unpredictable and if specific neuromuscular monitoring is not routinely employed it may result in the many complications associated with residual curarisation in the postoperative period. Residual curarisation may result in weakness, diplopia, hypoxaemia, atelectasis, aspiration and pneumonia.

It is generally accepted that receptor occupancy of $75 \%$ will result in a decrease in muscle function. Residual block can only reliably be detected with the use of a nerve stimulator, and a train of four ratio (TOFR) of greater than 0.9 (as measured at the adductor pollicis) is regarded as adequate reversal. The clinical methods used are unreliable and will indicate a reversal at a TOF ratio of $0.4-0.5$. A value of less than 0.7 was used up until the early 1990 s which means that studies done prior to this time underestimated the real incidence of residual curarisation in the postoperative period. ${ }^{18}$

A landmark study by Viby-Morgensen ${ }^{19}$ in 1979 showed that $42 \%$ of patients receiving a long-acting NDMBA had residual block with TOF ratio less than 0.7 on arrival in the post-anaesthesia care unit (PACU). Other studies in the 1980s showed figures of $21-36 \%$ had TOF ratios less than 0.7 in the PACU. A meta-analysis by Naguib et al..$^{20}$ in 2007 looking at 24 trials ( 3300 patients) that used TOF ratios greater than 0.9 showed that $41 \%$ of patients had residual block in the PACU after receiving an intermediate acting NDMBA. The incidence of this complication does not seem to be decreasing over time. The more widespread introduction of sugammadex into routine practice will certainly decrease the incidence of PORC but this is unlikely in the near future due to the much higher cost as compared to a neostigmine reversal.

A number of studies have been done in volunteers looking at the consequences of residual block with a TOF ratio less than 0.9. Awake volunteers with a TOF ratio of less than 0.9 may exhibit the following:

- impaired hypoxic ventilation responses;

- upper airway obstruction;

- impaired ability to swallow;

- impaired coordination of pharyngeal muscles;

- reduced upper oesophageal muscle tone increasing the risk of aspiration;

- all the volunteers had unpleasant symptoms of weakness.

These consequences are more severe in the patient with impaired physiologic reserve. There are a number of reasons why, 70 years after the introduction of Curare, residual curarisation remains a significant problem.
1. Doctors do not believe that it is a problem!

2. Incorrect and misleading measures of adequate reversal are performed.

2.1 Use of eye muscles - correlates with reversal of the diaphragm but not pharyngeal muscles. Adductor pollicis muscle correlates with reversal of respiratory muscles;

2.2 Direct muscle stimulation;

2.3 Looking at responses of fingers other than the thumb;

2.4 Visual or tactile assessment of fade. Once TOF ratio reaches $0.3-0.4$, fade cannot be detected reliably;

2.5 Clinical tests such as head lift and tidal volume are not sensitive enough. Volunteers were able to do a 5 second head lift with a TOF ratio of 0.6. Neostigmine has a ceiling effect so that if receptor occupancy is high it will not reverse the NDMBA and it has its peak effect in 10-15 minutes. Neostigmine should only be given when there are at least two to three twitches, and preferably four on the TOF. If given when there are 1-2 twitches, a TOF ratio of $0.4-0.7$ is quickly achieved with the appearance of no fade. A long plateau phase of recovery then ensues until complete reversal is obtained. The average time for reversal of a moderate neuromuscular block (TOFC $=1-3$ ) is 17.9 minutes after administration of $50 \mu \mathrm{g} . \mathrm{kg}-1$ neostigmine. Due to the ceiling effect of neostigmine, doses greater than $70 \mu \mathrm{g} / \mathrm{kg}$ or repeat doses should not be administered.

3. The perception that the problem does not occur with the newer intermediate acting NDMBAs. In Europe 20\% of anaesthetists as well as $10 \%$ of US, Australian and New Zealand anaesthetists never use nerve stimulators to guide management of NDMBAs. ${ }^{21}$ A European study looked at spontaneous reversal of vecuronium and rocuronium given at doses of 2X ED95. After 2 hours, $37 \%$ of patients had a TOF ratio of less than 0.9 and $10 \%$ a TOF ratio of less than $0.7 .^{18}$ This implies that a NDMBA should always be reversed if a nerve stimulator is not available. The issue of neostigmine causing muscle weakness in a patient that is fully reversed is only of importance if high doses of neostigmine $(>50-70 \mu \mathrm{g} / \mathrm{Kg}$ ) are used. The safest and most accurate measure is to use an appropriate quantitative neuromuscular monitor that can measure a TOF ratio.

\section{Sugammadex}

Sugammadex is a modified gamma-cyclodextrin that selectively binds and encapsulates vecuronium and rocuronium in a 1:1 ratio that is not reversible and renders the muscle relaxant inactive. The sugammadex/rocuronium or vecuronium compound is eliminated unchanged by the kidneys, with more than $90 \%$ being excreted in 24 hours. As the free muscle relaxant in plasma is 'mopped' up by sugammadex the gradient from receptor to plasma increases and so more relaxant leaves the receptor site and moves into the plasma to be bound by sugammadex. 
This rapidly restores muscle function. A higher sugammadex dose is thus more effective in 'mopping' up free drug in the plasma and establishing a higher concentration gradient from effector site to plasma. The dose of sugammadex therefore needs to be tailored to the degree of neuromuscular block. Reversing a deep block will require a larger dose of sugammadex otherwise residual neuromuscular block will occur.

Three dosing regimens are recommended depending on the degree of block. This makes it mandatory to monitor the depth of the NMB. A moderate block (TOFC $=2$ ) requires a dose of $2 \mathrm{mg} /$ $\mathrm{Kg}$. A deep block with a post-tetanic count $(\mathrm{PTC}=1-2)$ requires $4 \mathrm{mg} / \mathrm{Kg}$ whereas an intense block $(\mathrm{PTC}=0)$ requires $16 \mathrm{mg} / \mathrm{Kg}{ }^{1}$ Appropriate dosing according to the depth of block is important to prevent residual curarisation.

Sugammadex is unlikely to replace neostigmine as the reversal agent of choice in the near future. Cost constraints will for the foreseeable future limit the use of sugammadex to very specific indications. To reduce the incidence of postoperative residual block with the use of NDMBA and reversal by neostigmine, anaesthesiologists need to be educated in the use of a TOF ratio monitor so that neostigmine can be given at the appropriate time and in so doing avoid the dangerous complications that may arise when sending a patient to the PACU who still has a unsuspected residual neuromuscular block. The use of an appropriate neuromuscular monitor and preferably a quantitative neuromuscular monitor should be part of the basic minimum standards of monitoring in all patients who have been given a NDMBA.

\section{References:}

1. Welch EH. Neuromuscular blocking agents. In: Milner A, Welch EH, eds. Applied Pharmacology in Anaesthesia and Critical Care. Centurion, South Africa: Medpharm Publication (PYT) LTD; 2012:485-525.

2. Naguib M, Kopman AF, Lien CA, Hunter JM, Lopez A, Brull SJ. A survey of current management of neuromuscular block in the United States and Europe. Anesth Analg. 2010;111:110-9.

3. Ledowski T, Ong JS, Flett T. Neuromuscular monitoring, muscle relaxant use, and reversal at a tertiary teaching hospital 2.5 years after introduction of sugammadex: changes in opinions and clinical practice. Anesthesiol Res Pract. 2015;2015.

4. Rosenberg J, Herring WJ, Blobner M, et al. Deep neuromuscular blockade improves laparoscopic surgical conditions: a randomized, controlled study. Adv Ther. 2017;34:925-36
5. Shafer SL. Truth is elusive. Acta Anaesthesiol Scand. 2016;60:691-2.

6. Madsen M, Staehr-Rye A, Claudius C, Gätke M. Is deep neuromuscular blockade beneficial in laparoscopic surgery? Yes, probably. Acta Anaesthesiol Scand. 2016;60:710-6.

7. Kopman AF, Naguib M. Is deep neuromuscular block beneficial in laparoscopic surgery? No, probably not. Acta Anaesthesiol Scand. 2016;60:717-22.

8. Martini C, Boon M, Bevers R, Aarts L, Dahan A. Evaluation of surgical conditions during laparoscopic surgery in patients with moderate vs deep neuromuscular block. Br J Anaesth. 2013;112:498-505.

9. Blobner M, Frick CG, Stäuble RB, et al. Neuromuscular blockade improves surgical conditions (NISCO). Surg Endosc. 2015;29:627-36.

10. Staehr-Rye AK, Rasmussen LS, Rosenberg J, et al. Surgical space conditions during low-pressure laparoscopic cholecystectomy with deep versus moderate neuromuscular blockade: a randomized clinical study. Anesth Analg. 2014;119:1084-92.

11. Madsen MV, Istre 0 , Staehr-Rye AK, et al. Postoperative shoulder pain after laparoscopic hysterectomy with deep neuromuscular blockade and low-pressure pneumoperitoneum: a randomised controlled trial. Eur J Anaesthesiol (EJA). 2016;33:341-7.

12. Neudecker J, Sauerland S, Neugebauer E, et al. The European Association for Endoscopic Surgery clinical practice guideline on the pneumoperitoneum for laparoscopic surgery. Surg Endosc. 2002;16:1121-43.

13. Gurusamy KS, Vaughan J, Davidson BR. Low pressure versus standard pressure pneumoperitoneum in laparoscopic cholecystectomy. The Cochrane Library. 2014.

14. Dubois PE, Putz L, Jamart J, Marotta M-L, Gourdin M, Donnez O. Deep neuromuscular block improves surgical conditions during laparoscopic hysterectomy: a randomised controlled trial. Eur J Anaesthesiol (EJA). 2014:31:430-6.

15. Boon M, Martini C, Dahan A. Recent advances in neuromuscular block during anesthesia. F1000Research. 2018;7.

16. Ezri T, Evron S, Petrov I, Schachter P, Berlovitz Y, Shimonov M. Residual curarization and postoperative respiratory complications following laparoscopic sleeve gastrectomy. The effect of reversal agents: sugammadex vs. neostigmine. J Crit Care Med. 2015;1:61-7.

17. Della Rocca G, Pompei L, Pagan De Paganis C, et al. Reversal of rocuronium induced neuromuscular block with sugammadex or neostigmine: a large observational study. Acta Anaesthesiol Scand. 2013;57:1138-45.

18. Debaene B, Plaud B, Dilly M-P, Donati F. Residual paralysis in the PACU after a single intubating dose of nondepolarizing muscle relaxant with an intermediate duration of action. Anesthesiology. 2003;98:1042-8.

19. Viby-Mogensen J, Jørgensen BC, Ørding H. Residual curarization in the recovery room. Anesthesiology. 1979;50:539-41.

20. Naguib MLC, Meistelman C. Pharmacology of Neuromuscular drugs. In: D MR, Millers, ed. Anesthesia. 8 ed; p. 958-94.

21. Brull SJ, Kopman AF. Current Status of Neuromuscular Reversal and MonitoringChallenges and Opportunities. Anesthesiology. 2017;126:173-90. 